\title{
On complete submanifolds with parallel mean curvature in product spaces
}

\author{
Dorel Fetcu and Harold Rosenberg
}

\begin{abstract}
We prove a Simons type formula for submanifolds with parallel mean curvature vector field in product spaces of type $M^{n}(c) \times \mathbb{R}$, where $M^{n}(c)$ is a space form with constant sectional curvature $c$, and then we use it to characterize some of these submanifolds.
\end{abstract}

\section{Introduction}

In 1968, James Simons obtained an equation for the Laplacian of the second fundamental form of a minimal submanifold of a Riemannian manifold (see [18]). He then applied this theorem in several ways; in particular by characterizing certain minimal submanifolds of spheres. Over the years, such formulas, now called Simons type equations, proved to be a powerful tool not only for studying minimal submanifolds in Riemannian manifolds, but also, more generally, for studying submanifolds with constant mean curvature (cmc submanifolds) or with parallel mean curvature vector (pmc submanifolds). Special attention was paid to $\mathrm{cmc}$ and pmc submanifolds in space forms, articles like [2], [5], [8], [10], [15], [16], [17], [20] being only a few examples of contributions on this topic in which Simons type formulas are used to prove gap and reduction of codimension theorems. An excellent presentation of the classical result of Simons and some of its applications can be found in the very recent book [9]. The authors point out, for example, how Simons' equation can be used to obtain curvature bounds for minimal surfaces with small total curvature and also curvature estimates for stable minimal surfaces in $\mathbb{R}^{3}$, and then, more generally, for stable minimal hypersurfaces in $\mathbb{R}^{n}$.

Recently, such equations were obtained for $\mathrm{cmc}$ and pmc submanifolds in product spaces of the form $M^{n}(c) \times \mathbb{R}$, where $M^{n}(c)$ stands for an $n$-dimensional space form with constant sectional curvature $c$, and then used to characterize some of these submanifolds (see, for example, [6], [12]). More exactly, in [6] the author computed the Laplacian of the second fundamental form of a cmc surface in $M^{3}(c) \times \mathbb{R}$,

Mathematics Subject Classification (2010): Primary 53C42; Secondary 53A10.

Keywords: Submanifolds with parallel mean curvature vector field, Simons type equation. 
as well as the Laplacian of the traceless part of the Abresch-Rosenberg differential introduced in [1] for such surfaces, whilst in [12] there was found the expression of the Laplacian of $\left|A_{H}\right|^{2}$ for a pmc submanifold in $M^{n}(c) \times \mathbb{R}$ with shape operator $A$ and mean curvature vector field $H$.

In our paper, we first compute the Laplacian of the second fundamental form of a pmc submanifold in $M^{n}(c) \times \mathbb{R}$ and then we use this Simons type formula to prove some gap theorems for pmc submanifolds in $M^{n}(c) \times \mathbb{R}$ when $c>0$ and the mean curvature vector field $H$ of the submanifold makes a constant angle with the unit vector field $\xi$ tangent to $\mathbb{R}$, or when $c<0$ and $H$ is orthogonal to $\xi$.

Our main results are the following four theorems.

Theorem 4.4. Let $\Sigma^{m}$ be an immersed complete non-minimal pmc submanifold in $M^{n}(c) \times \mathbb{R}, n>m \geq 3, c>0$, with mean curvature vector field $H$ and second fundamental form $\sigma$. If the angle between $H$ and $\xi$ is constant and

$$
|\sigma|^{2}+\frac{2 c(2 m+1)}{m}|T|^{2} \leq 2 c+\frac{m^{2}}{m-1}|H|^{2},
$$

where $T$ is the tangent part of $\xi$, then $\Sigma^{m}$ is a totally umbilical cmc hypersurface in $M^{m+1}(c)$.

Theorem 4.5. Let $\Sigma^{m}$ be an immersed complete non-minimal pmc submanifold in $M^{n}(c) \times \mathbb{R}, n>m \geq 3, c<0$, with mean curvature vector field $H$ and second fundamental form $\sigma$. If $H$ is orthogonal to $\xi$ and

$$
|\sigma|^{2}+\frac{2 c(m+1)}{m}|T|^{2} \leq 4 c+\frac{m^{2}}{m-1}|H|^{2},
$$

then $\Sigma^{m}$ is a totally umbilical cmc hypersurface in $M^{m+1}(c)$.

Theorem 4.6. Let $\Sigma^{2}$ be a complete non-minimal pmc surface in $M^{n}(c) \times \mathbb{R}$, $n>2, c>0$, such that the angle between $H$ and $\xi$ is constant and

$$
|\sigma|^{2}+3 c|T|^{2} \leq 4|H|^{2}+2 c .
$$

Then, either

1. $\Sigma^{2}$ is pseudo-umbilical and lies in $M^{n}(c)$; or

2. $\Sigma^{2}$ is a torus $\mathbb{S}^{1}(r) \times \mathbb{S}^{1}\left(\sqrt{1 / c-r^{2}}\right)$ in $M^{3}(c)$, with $r^{2} \neq 1 /(2 c)$.

Theorem 4.7. Let $\Sigma^{2}$ be a complete non-minimal pmc surface in $M^{n}(c) \times \mathbb{R}$, $n>2, c<0$, such that $H$ is orthogonal to $\xi$ and

$$
|\sigma|^{2}+5 c|T|^{2} \leq 4|H|^{2}+4 c .
$$

Then $\Sigma^{2}$ is pseudo-umbilical and lies in $M^{n}(c)$.

Acknowledgments. The first author would like to thank the IMPA in Rio de Janeiro for providing a very stimulating work environment during the preparation of this paper. 


\section{Preliminaries}

Let $M^{n}(c)$ be a space form, i.e., a simply-connected $n$-dimensional manifold with constant sectional curvature $c$. Thus, $M^{n}(c)$ is the sphere $\mathbb{S}^{n}(c)$, the Euclidean space, or the hyperbolic space $\mathbb{H}^{n}(c)$, as $c>0, c=0$, or $c<0$. Now, let us consider the product manifold $\bar{M}=M^{n}(c) \times \mathbb{R}$. The expression of the curvature tensor $\bar{R}$ of such a manifold can be obtained from

$$
\langle\bar{R}(X, Y) Z, W\rangle=c\{\langle d \pi Y, d \pi Z\rangle\langle d \pi X, d \pi W\rangle-\langle d \pi X, d \pi Z\rangle\langle d \pi Y, d \pi W\rangle\},
$$

where $\pi: \bar{M}=M^{n}(c) \times \mathbb{R} \rightarrow M^{n}(c)$ is the projection map. After a straightforward computation we get

$$
\begin{aligned}
\bar{R}(X, Y) Z= & c\{\langle Y, Z\rangle X-\langle X, Z\rangle Y-\langle Y, \xi\rangle\langle Z, \xi\rangle X+\langle X, \xi\rangle\langle Z, \xi\rangle Y \\
& +\langle X, Z\rangle\langle Y, \xi\rangle \xi-\langle Y, Z\rangle\langle X, \xi\rangle \xi\},
\end{aligned}
$$

where $\xi$ is the unit vector tangent to $\mathbb{R}$.

Let $\Sigma^{m}$ be an $m$-dimensional submanifold of $\bar{M}$. From the Gauss equation

$$
\begin{aligned}
\langle R(X, Y) Z, W\rangle= & \langle\bar{R}(X, Y) Z, W\rangle \\
& +\sum_{\alpha=m+1}^{n+1}\left\{\left\langle A_{\alpha} Y, Z\right\rangle\left\langle A_{\alpha} X, W\right\rangle-\left\langle A_{\alpha} X, Z\right\rangle\left\langle A_{\alpha} Y, W\right\rangle\right\},
\end{aligned}
$$

we obtain the expression of its curvature tensor,

$$
\begin{aligned}
R(X, Y) Z=c & \{Y, Z\rangle X-\langle X, Z\rangle Y-\langle Y, T\rangle\langle Z, T\rangle X+\langle X, T\rangle\langle Z, T\rangle Y \\
& +\langle X, Z\rangle\langle Y, T\rangle T-\langle Y, Z\rangle\langle X, T\rangle T\} \\
& +\sum_{\alpha=m+1}^{n+1}\left\{\left\langle A_{\alpha} Y, Z\right\rangle A_{\alpha} X-\left\langle A_{\alpha} X, Z\right\rangle A_{\alpha} Y\right\},
\end{aligned}
$$

where $T$ is the component of $\xi$ tangent to $\Sigma^{m}$ and $A$ is the shape operator defined by the Weingarten equation

$$
\bar{\nabla}_{X} V=-A_{V} X+\nabla_{X}^{\perp} V
$$

for any vector field $X$ tangent to $\Sigma^{m}$ and any normal vector field $V$. Here $\bar{\nabla}$ is the Levi-Civita connection on $\bar{M}, \nabla^{\perp}$ is the connection in the normal bundle, and $A_{\alpha}=A_{E_{\alpha}}$, where $\left\{E_{\alpha}\right\}_{\alpha=m+1}^{n+1}$ is a local orthonormal frame field in the normal bundle.

Definition 2.1. A submanifold $\Sigma^{m}$ of $M^{n}(c) \times \mathbb{R}$ is called a vertical cylinder over $\Sigma^{m-1}$ if $\Sigma^{m}=\pi^{-1}\left(\Sigma^{m-1}\right)$, where $\pi: M^{n}(c) \times \mathbb{R} \rightarrow M^{n}(c)$ is the projection map and $\Sigma^{m-1}$ is a submanifold of $M^{n}(c)$.

It is easy to see that vertical cylinders $\Sigma^{m}=\pi^{-1}\left(\Sigma^{m-1}\right)$ are characterized by the fact that $\xi$ is tangent to $\Sigma^{m}$.

Definition 2.2. If the mean curvature vector field $H$ of a submanifold $\Sigma^{m}$ is parallel in the normal bundle, i.e., $\nabla^{\perp} H=0$, then $\Sigma^{m}$ is called a pmc submanifold. 
Remark 2.3. It is straightforward to verify that $\Sigma^{m}=\pi^{-1}\left(\Sigma^{m-1}\right)$ is a pmc vertical cylinder in $M^{n}(c) \times \mathbb{R}$ if and only if $\Sigma^{m-1}$ is a pmc submanifold in $M^{n}(c)$. Moreover, the mean curvature vector field of $\Sigma^{m}$ is $H=\frac{m-1}{m} H_{0}$, where $H_{0}$ is the mean curvature vector field of $\Sigma^{m-1}$.

We end this section by recalling the following three results, which we shall use later in this paper.

Lemma $2.4([7])$. Let $a_{1}, \ldots, a_{m}$, where $m>1$, and $b$ be real numbers such that

$$
\left(\sum_{i=1}^{n} a_{i}\right)^{2} \geq(n-1) \sum_{i=1}^{n} a_{i}^{2}+b
$$

Then, for all $i \neq j$, we have

$$
2 a_{i} a_{j} \geq \frac{b}{n-1}
$$

Moreover, if the inequality (2.3) is strict, then so are the inequalities (2.4).

Lemma 2.5 ([14]). Let $A_{1}, \ldots, A_{p}$, where $p \geq 2$, be symmetric $m \times m$ matrices. Then

$$
\sum_{\alpha, \beta=1}^{p}\left\{N\left(A_{\alpha} A_{\beta}-A_{\beta} A_{\alpha}\right)+\left(\operatorname{trace}\left(A_{\alpha} A_{\beta}\right)\right)^{2}\right\} \leq \frac{3}{2}\left(\sum_{\alpha=1}^{p} N\left(A_{\alpha}\right)\right)^{2},
$$

where $N(A)=\operatorname{trace}\left(A^{t} A\right)$. Equality holds if and only if either

1) $A_{1}=\cdots=A_{p}=0$; or

2) only two matrices $A_{\alpha_{0}}$ and $A_{\beta_{0}}$ are different from the null $m \times m$ matrix. Moreover, in this case, $N\left(A_{\alpha_{0}}\right)=N\left(A_{\beta_{0}}\right)=L$ and there exists an orthogonal matrix $T$ such that

$$
T^{t} A_{\alpha_{0}} T=\sqrt{\frac{L}{2}}\left(\begin{array}{ccccc}
1 & 0 & 0 & \cdots & 0 \\
0 & -1 & 0 & \cdots & 0 \\
0 & 0 & 0 & \cdots & 0 \\
\vdots & \vdots & \vdots & \ddots & \vdots \\
0 & 0 & 0 & \cdots & 0
\end{array}\right) \text { and } T^{t} A_{\beta_{0}} T=\sqrt{\frac{L}{2}}\left(\begin{array}{ccccc}
0 & 1 & 0 & \cdots & 0 \\
1 & 0 & 0 & \cdots & 0 \\
0 & 0 & 0 & \cdots & 0 \\
\vdots & \vdots & \vdots & \ddots & \vdots \\
0 & 0 & 0 & \cdots & 0
\end{array}\right) \text {. }
$$

Theorem 2.6 (Omori-Yau maximum principle, [21]). If $\Sigma^{m}$ is a complete Riemannian manifold with Ricci curvature bounded from below, then for any smooth function $u \in C^{2}\left(\Sigma^{m}\right)$ with $\sup _{\Sigma^{m}} u<+\infty$ there exists a sequence of points $\left\{p_{k}\right\}_{k \in \mathbb{N}} \subset \Sigma^{m}$ satisfying

$$
\lim _{k \rightarrow \infty} u\left(p_{k}\right)=\sup _{\Sigma^{m}} u, \quad|\nabla u|\left(p_{k}\right)<\frac{1}{k} \quad \text { and } \quad \Delta u\left(p_{k}\right)<\frac{1}{k} .
$$




\section{A Simons type formula for pmc submanifolds in $M^{n}(c) \times \mathbb{R}$}

Let $\Sigma^{m}$ be an $m$-dimensional pmc submanifold in $M^{n}(c) \times \mathbb{R}$ with mean curvature vector field $H$.

In the following, we shall compute the Laplacian of the squared norm of the second fundamental form $\sigma$ of $\Sigma^{m}$, where $\sigma$ is defined by the Gauss equation

$$
\bar{\nabla}_{X} Y=\nabla_{X} Y+\sigma(X, Y)
$$

for any tangent vector fields $X$ and $Y$.

Let $\left\{E_{m+1}, \ldots, E_{n+1}\right\}$ be a local orthonormal frame field in the normal bundle. Then, normal connection forms $s_{\alpha \beta}$ are determined by

$$
\nabla_{X}^{\perp} E_{\alpha}=\sum_{\beta=m+1}^{n+1} s_{\alpha \beta}(X) E_{\beta}
$$

for any vector field $X$ tangent to $\Sigma^{n}$ and any $\alpha \in\{m+1, \ldots, n+1\}$. It is easy to see that $s_{\alpha \beta}=-s_{\beta \alpha}$ and that

$$
\begin{aligned}
\nabla_{X}^{\perp} H & =\frac{1}{m} \nabla_{X}^{\perp}(\operatorname{trace} \sigma)=\frac{1}{m} \sum_{\alpha=m+1}^{n+1} \nabla_{X}^{\perp}\left(\left(\operatorname{trace} A_{\alpha}\right) E_{\alpha}\right) \\
& =\frac{1}{m} \sum_{\alpha=m+1}^{n+1}\left(X\left(\operatorname{trace} A_{\alpha}\right)-\sum_{\beta=m+1}^{n+1} s_{\alpha \beta}(X) \operatorname{trace} A_{\beta}\right) E_{\alpha}
\end{aligned}
$$

Therefore, the mean curvature vector field $H$ is parallel if and only if

$$
X\left(\operatorname{trace} A_{\alpha}\right)-\sum_{\beta=m+1}^{n+1} s_{\alpha \beta}(X) \operatorname{trace} A_{\beta}=0
$$

for all $\alpha$ 's.

Now, from the Codazzi equation,

$$
\begin{aligned}
\left\langle\bar{R}(X, Y) Z, E_{\alpha}\right\rangle= & \left\langle\nabla_{X}^{\perp} \sigma(Y, Z), E_{\alpha}\right\rangle-\left\langle\sigma\left(\nabla_{X} Y, Z\right), E_{\alpha}\right\rangle-\left\langle\sigma\left(Y, \nabla_{X} Z\right), E_{\alpha}\right\rangle \\
& -\left\langle\nabla_{Y}^{\perp} \sigma(X, Z), E_{\alpha}\right\rangle+\left\langle\sigma\left(\nabla_{Y} X, Z\right), E_{\alpha}\right\rangle+\left\langle\sigma\left(X, \nabla_{Y} Z\right), E_{\alpha}\right\rangle,
\end{aligned}
$$

we get

$$
\begin{aligned}
\left\langle\bar{R}(X, Y) Z, E_{\alpha}\right\rangle= & X\left(\left\langle A_{\alpha} Y, Z\right\rangle\right)-\left\langle\sigma(Y, Z), \nabla_{X}^{\perp} E_{\alpha}\right\rangle-\left\langle A_{\alpha}\left(\nabla_{X} Y\right), Z\right\rangle \\
& -\left\langle A_{\alpha} Y, \nabla_{X} Z\right\rangle-Y\left(\left\langle A_{\alpha} X, Z\right\rangle\right)+\left\langle\sigma(X, Z), \nabla_{Y}^{\perp} E_{\alpha}\right\rangle \\
& +\left\langle A_{\alpha}\left(\nabla_{Y} X\right), Z\right\rangle+\left\langle A_{\alpha} X, \nabla_{Y} Z\right\rangle \\
= & \left\langle\left(\nabla_{X} A_{\alpha}\right) Y-\left(\nabla_{Y} A_{\alpha}\right) X, Z\right\rangle \\
& -\sum_{\beta=m+1}^{n+1}\left\langle s_{\alpha \beta}(X) A_{\beta} Y-s_{\alpha \beta}(Y) A_{\beta} X, Z\right\rangle .
\end{aligned}
$$


Therefore, using (2.1), we obtain

$$
\begin{aligned}
\left(\nabla_{X} A_{\alpha}\right) Y= & \left(\nabla_{Y} A_{\alpha}\right) X+\sum_{\beta=m+1}^{n+1}\left(s_{\alpha \beta}(X) A_{\beta} Y-s_{\alpha \beta}(Y) A_{\beta} X\right) \\
& +c\left\langle E_{\alpha}, N\right\rangle(\langle Y, T\rangle X-\langle X, T\rangle Y)
\end{aligned}
$$

where $N$ is the normal part of $\xi$.

Next, we have the following Weitzenböck formula:

$$
\frac{1}{2} \Delta\left|A_{\alpha}\right|^{2}=\left|\nabla A_{\alpha}\right|^{2}+\left\langle\operatorname{trace} \nabla^{2} A_{\alpha}, A_{\alpha}\right\rangle,
$$

where we have extended the metric $\langle$,$\rangle to the tensor space in the standard way.$

The second term on the right hand side of (3.3) can be calculated by using a method introduced in [16] and developed in [10].

Let us consider

$$
C_{\alpha}(X, Y)=\left(\nabla^{2} A_{\alpha}\right)(X, Y)=\nabla_{X}\left(\nabla_{Y} A_{\alpha}\right)-\nabla_{\nabla_{X} Y} A_{\alpha},
$$

and note that we have the following Ricci commutation formula:

$$
C_{\alpha}(X, Y)=C_{\alpha}(Y, X)+\left[R(X, Y), A_{\alpha}\right] .
$$

Next, consider an orthonormal basis $\left\{e_{i}\right\}_{i=1}^{m}$ in $T_{p} \Sigma^{m}, p \in \Sigma^{m}$, extend $e_{i}$ to vector fields $E_{i}$ in a neighborhood of $p$ such that $\left\{E_{i}\right\}$ is a geodesic frame field around $p$, and write $X=E_{k}$. We have

$$
\left(\operatorname{trace} \nabla^{2} A_{\alpha}\right) X=\sum_{i=1}^{m} C_{\alpha}\left(E_{i}, E_{i}\right) X .
$$

Using equation (3.2), we get, at $p$,

$$
\begin{aligned}
C_{\alpha}\left(E_{i}, X\right) E_{i}= & \nabla_{E_{i}}\left(\left(\nabla_{X} A_{\alpha}\right) E_{i}\right) \\
= & \nabla_{E_{i}}\left(\left(\nabla_{E_{i}} A_{\alpha}\right) X\right)+\nabla_{E_{i}}\left(\sum_{\beta=m+1}^{n+1}\left(s_{\alpha \beta}(X) A_{\beta} E_{i}-s_{\alpha \beta}\left(E_{i}\right) A_{\beta} X\right)\right) \\
& +c \nabla_{E_{i}}\left(\left\langle E_{\alpha}, N\right\rangle\left(\left\langle E_{i}, T\right\rangle X-\langle X, T\rangle E_{i}\right)\right)
\end{aligned}
$$

and then

$$
\begin{aligned}
C_{\alpha}\left(E_{i}, X\right) E_{i}= & C_{\alpha}\left(E_{i}, E_{i}\right) X+\nabla_{E_{i}}\left(\sum_{\beta=m+1}^{n+1}\left(s_{\alpha \beta}(X) A_{\beta} E_{i}-s_{\alpha \beta}\left(E_{i}\right) A_{\beta} X\right)\right) \\
& +c \sum_{\beta=m+1}^{n+1}\left\langle s_{\alpha \beta}\left(E_{i}\right) E_{\beta}, N\right\rangle\left(\left\langle E_{i}, T\right\rangle X-\langle X, T\rangle E_{i}\right) \\
& -c\left\langle A_{\alpha} E_{i}, T\right\rangle\left(\left\langle E_{i}, T\right\rangle X-\langle X, T\rangle E_{i}\right) \\
& +c\left\langle E_{\alpha}, N\right\rangle\left(\left\langle A_{N} E_{i}, E_{i}\right\rangle X-\left\langle A_{N} X, E_{i}\right\rangle E_{i}\right)
\end{aligned}
$$


where we used $\sigma\left(E_{i}, T\right)=-\nabla_{E_{i}}^{\perp} N$ and $\nabla_{E_{i}} T=A_{N} E_{i}$, which follow from the fact that $\xi$ is parallel, i.e., $\bar{\nabla} \xi=0$.

We also have, at $p$,

$$
C_{\alpha}\left(X, E_{i}\right) E_{i}=\nabla_{X}\left(\left(\nabla_{E_{i}} A_{\alpha}\right) E_{i}\right)
$$

and then, from (3.4), (3.5) and (3.6), we get

$$
\begin{aligned}
& C_{\alpha}\left(E_{i}, E_{i}\right) X=\nabla_{X}\left(\left(\nabla_{E_{i}} A_{\alpha}\right) E_{i}\right)+\left[R\left(E_{i}, X\right), A_{\alpha}\right] E_{i} \\
& \quad-\nabla_{E_{i}}\left(\sum_{\beta=m+1}^{n+1}\left(s_{\alpha \beta}(X) A_{\beta} E_{i}-s_{\alpha \beta}\left(E_{i}\right) A_{\beta} X\right)\right) \\
& \quad-c \sum_{\beta=m+1}^{n+1}\left\langle s_{\alpha \beta}\left(E_{i}\right) E_{\beta}, N\right\rangle\left(\left\langle E_{i}, T\right\rangle X-\langle X, T\rangle E_{i}\right) \\
& \quad+c\left\langle A_{\alpha} E_{i}, T\right\rangle\left(\left\langle E_{i}, T\right\rangle X-\langle X, T\rangle E_{i}\right)-c\left\langle E_{\alpha}, N\right\rangle\left(\left\langle A_{N} E_{i}, E_{i}\right\rangle X-\left\langle A_{N} X, E_{i}\right\rangle E_{i}\right) .
\end{aligned}
$$

Since $\nabla_{E_{i}} A_{\alpha}$ is symmetric, from (3.2) one obtains

$$
\begin{aligned}
& \left\langle\sum_{i=1}^{m}\left(\nabla_{E_{i}} A_{\alpha}\right) E_{i}, Z\right\rangle=\sum_{i=1}^{m}\left\langle E_{i},\left(\nabla_{E_{i}} A_{\alpha}\right) Z\right\rangle \\
(3.7)= & \sum_{i=1}^{m}\left\langle E_{i},\left(\nabla_{Z} A_{\alpha}\right) E_{i}\right\rangle-\sum_{i=1}^{m}\left\langle\sum_{\beta=m+1}^{n+1} s_{\alpha \beta}(Z) A_{\beta} E_{i}, E_{i}\right\rangle \\
& +\sum_{i=1}^{m}\left\langle\sum_{\beta=m+1}^{n+1} s_{\alpha \beta}\left(E_{i}\right) A_{\beta} E_{i}, Z\right\rangle+c\left\langle E_{\alpha}, N\right\rangle \sum_{i=1}^{m}\left\langle E_{i},\langle Z, T\rangle E_{i}-\left\langle E_{i}, T\right\rangle Z\right\rangle,
\end{aligned}
$$

which, together with (3.1), leads to

$$
\begin{aligned}
\left\langle\sum_{i=1}^{m}\left(\nabla_{E_{i}} A_{\alpha}\right) E_{i}, Z\right\rangle= & Z\left(\operatorname{trace} A_{\alpha}\right)-\sum_{\beta=m+1}^{n+1} s_{\alpha \beta}(Z) \operatorname{trace} A_{\beta} \\
& +\left\langle\sum_{i=1}^{m} \sum_{\beta=m+1}^{n+1} s_{\alpha \beta}\left(E_{i}\right) A_{\beta} E_{i}+c(m-1)\left\langle E_{\alpha}, N\right\rangle T, Z\right\rangle \\
= & \left\langle\sum_{i=1}^{m} \sum_{\beta=m+1}^{n+1} s_{\alpha \beta}\left(E_{i}\right) A_{\beta} E_{i}+c(m-1)\left\langle E_{\alpha}, N\right\rangle T, Z\right\rangle
\end{aligned}
$$

for any vector $Z$ tangent to $\Sigma^{m}$. 
Therefore, we have

$$
\begin{aligned}
&\left(\operatorname{trace} \nabla^{2} A_{\alpha}\right) X=\sum_{i=1}^{m} C_{\alpha}\left(E_{i}, E_{i}\right) X \\
&=\sum_{i=1}^{m} \sum_{\beta=m+1}^{n+1}\left\{X\left(s_{\alpha \beta}\left(E_{i}\right)\right) A_{\beta} E_{i}+s_{\alpha \beta}\left(E_{i}\right) \nabla_{X} A_{\beta} E_{i}\right. \\
&-E_{i}\left(s_{\alpha \beta}(X)\right) A_{\beta} E_{i}-s_{\alpha \beta}(X) \nabla_{E_{i}} A_{\beta} E_{i} \\
&\left.+E_{i}\left(s_{\alpha \beta}\left(E_{i}\right)\right) A_{\beta} X+s_{\alpha \beta}\left(E_{i}\right) \nabla_{E_{i}} A_{\beta} X\right\} \\
&+c(m-1)\left\langle\sum_{\beta=m+1}^{n+1} s_{\alpha \beta}(X) E_{\beta}, N\right\rangle T \\
&-c \sum_{i=1}^{m}\left\langle\sum_{\beta=m+1}^{n+1} s_{\alpha \beta}\left(E_{i}\right) E_{\beta}, N\right\rangle\left(\left\langle E_{i}, T\right\rangle X-\langle X, T\rangle E_{i}\right) \\
&+c\left\langle A_{\alpha} T, T\right\rangle X-c\langle X, T\rangle A_{\alpha} T-c m\left\langle E_{\alpha}, N\right\rangle\langle H, N\rangle X \\
&+c m\left\langle E_{\alpha}, N\right\rangle A_{N} X-c(m-1)\left\langle A_{\alpha} T, X\right\rangle T \\
&+\sum_{i=1}^{m}\left[R\left(E_{i}, X\right), A_{\alpha}\right] E_{i} .
\end{aligned}
$$

Now, using the Ricci equation

$$
\left\langle R^{\perp}(X, Y) E_{\alpha}, E_{\beta}\right\rangle=\left\langle\left[A_{\alpha}, A_{\beta}\right] X, Y\right\rangle+\left\langle\bar{R}(X, Y) E_{\alpha}, E_{\beta}\right\rangle,
$$

we get, after a straightforward computation,

$$
\begin{aligned}
& \sum_{i=1}^{m} \sum_{\beta=m+1}^{n+1}\left(X\left(s_{\alpha \beta}\left(E_{i}\right)\right) A_{\beta} E_{i}-E_{i}\left(s_{\alpha \beta}(X)\right) A_{\beta} E_{i}\right) \\
&=\sum_{i=1}^{m} \sum_{\beta=m+1}^{n+1}\left(\left(\nabla_{X} s_{\alpha \beta}\right)\left(E_{i}\right) A_{\beta} E_{i}-\left(\nabla_{E_{i}} s_{\alpha \beta}\right)(X) A_{\beta} E_{i}\right) \\
&=\sum_{\beta=m+1}^{n+1} A_{\beta}\left[A_{\alpha}, A_{\beta}\right] X-\sum_{i=1}^{m} \sum_{\beta, \gamma=m+1}^{n+1} s_{\alpha \gamma}\left(E_{i}\right) s_{\gamma \beta}(X) A_{\beta} E_{i} \\
&+\sum_{i=1}^{m} \sum_{\beta, \gamma=m+1}^{n+1} s_{\alpha \gamma}(X) s_{\gamma \beta}\left(E_{i}\right) A_{\beta} E_{i} .
\end{aligned}
$$


From (3.2), we have

$$
\begin{aligned}
\sum_{i=1}^{m} & \sum_{\beta=m+1}^{n+1} s_{\alpha \beta}\left(E_{i}\right) \nabla_{X} A_{\beta} E_{i}=\sum_{i=1}^{m} \sum_{\beta=m+1}^{n+1} s_{\alpha \beta}\left(E_{i}\right)\left(\nabla_{X} A_{\beta}\right) E_{i} \\
= & \sum_{i=1}^{m} \sum_{\beta=m+1}^{n+1} s_{\alpha \beta}\left(E_{i}\right)\left\{\left(\nabla_{E_{i}} A_{\beta}\right) X-c\left\langle E_{\beta}, N\right\rangle\left(\langle X, T\rangle E_{i}-\left\langle E_{i}, T\right\rangle X\right)\right. \\
& \left.\quad-\sum_{\gamma=m+1}^{n+1}\left(s_{\beta \gamma}\left(E_{i}\right) A_{\gamma} X-s_{\beta \gamma}(X) A_{\gamma} E_{i}\right)\right\} .
\end{aligned}
$$

We now use (3.8) to compute

$$
\begin{aligned}
& \sum_{i=1}^{m} \sum_{\beta=m+1}^{n+1} s_{\alpha \beta}(X) \nabla_{E_{i}} A_{\beta} E_{i}=\sum_{i=1}^{m} \sum_{\beta=m+1}^{n+1} s_{\alpha \beta}(X)\left(\nabla_{E_{i}} A_{\beta}\right) E_{i} \\
& =\sum_{i=1}^{m} \sum_{\beta, \gamma=m+1}^{n+1} s_{\alpha \beta}(X) s_{\beta \gamma}\left(E_{i}\right) A_{\gamma} E_{i} \\
& \quad+c(m-1)\left\langle\sum_{\beta=m+1}^{n+1} s_{\alpha \beta}(X) E_{\beta}, N\right\rangle T .
\end{aligned}
$$

From the Gauss equation (2.2) of $\Sigma^{m}$, we get

$$
\begin{aligned}
\sum_{i=1}^{m} R\left(E_{i}, X\right) A_{\alpha} E_{i}= & c\left\{A_{\alpha} X-\left(\operatorname{trace} A_{\alpha}\right) X+\left(\operatorname{trace} A_{\alpha}\right)\langle X, T\rangle T\right. \\
& \left.-\left\langle A_{\alpha} X, T\right\rangle T-\langle X, T\rangle A_{\alpha} T+\left\langle A_{\alpha} T, T\right\rangle X\right\} \\
& +\sum_{\beta=m+1}^{n+1}\left\{A_{\beta} A_{\alpha} A_{\beta} X-\left(\operatorname{trace}\left(A_{\alpha} A_{\beta}\right)\right) A_{\beta} X\right\}
\end{aligned}
$$

and

$$
\begin{aligned}
\sum_{i=1}^{m} A_{\alpha} R\left(E_{i}, X\right) E_{i}= & -c\left\{\left(m-1-|T|^{2}\right) A_{\alpha} X-(m-2)\langle X, T\rangle A_{\alpha} T\right\} \\
& +\sum_{\beta=m+1}^{n+1}\left\{A_{\alpha} A_{\beta}^{2} X-\left(\operatorname{trace} A_{\beta}\right) A_{\alpha} A_{\beta} X\right\} .
\end{aligned}
$$

Finally, taking into account that

$$
E_{i}\left(s_{\alpha \beta}\left(E_{i}\right)\right) A_{\beta} X=\left(\nabla_{E_{i}} s_{\alpha \beta}\right)\left(E_{i}\right) A_{\beta} X
$$

and then replacing $(3.10),(3.11),(3.12),(3.13)$ and (3.14) in (3.9), we obtain, after 
a long but straightforward computation,

$$
\begin{aligned}
& \left\langle\operatorname{trace} \nabla^{2} A_{\alpha}, A_{\alpha}\right\rangle=\sum_{i=1}^{m}\left\langle\left(\operatorname{trace} \nabla^{2} A_{\alpha}\right) E_{i}, A_{\alpha} E_{i}\right\rangle \\
& =\sum_{i=1}^{m}\left\{\sum_{\beta=m+1}^{n+1} 2 s_{\alpha \beta}\left(E_{i}\right) \operatorname{trace}\left(\left(\nabla_{E_{i}} A_{\beta}\right) A_{\alpha}\right)\right. \\
& \quad-\sum_{\beta, \gamma=m+1}^{n+1} s_{\alpha \gamma}\left(E_{i}\right) s_{\gamma \beta}\left(E_{i}\right) \operatorname{trace}\left(A_{\alpha} A_{\beta}\right) \\
& \left.\quad+\sum_{\beta=m+1}^{n+1}\left(\nabla_{E_{i}} s_{\alpha \beta}\right)\left(E_{i}\right) \operatorname{trace}\left(A_{\alpha} A_{\beta}\right)\right\} \\
& \quad+c\left\{\left(m-|T|^{2}\right)\left|A_{\alpha}\right|^{2}-2 m\left|A_{\alpha} T\right|^{2}+3\left(\operatorname{trace} A_{\alpha}\right)\left\langle A_{\alpha} T, T\right\rangle\right. \\
& \left.\quad+m\left(\operatorname{trace}\left(A_{N} A_{\alpha}\right)\right)\left\langle E_{\alpha}, N\right\rangle-\left(\operatorname{trace} A_{\alpha}\right)^{2}-m\left(\operatorname{trace} A_{\alpha}\right)\langle H, N\rangle\left\langle E_{\alpha}, N\right\rangle\right\} \\
& \quad+\sum_{\beta=m+1}^{n+1}\left\{\left(\operatorname{trace} A_{\beta}\right)\left(\operatorname{trace}\left(A_{\alpha}^{2} A_{\beta}\right)\right)+\operatorname{trace}\left[A_{\alpha}, A_{\beta}\right]^{2}\right. \\
& \left.\quad-\left(\operatorname{trace}\left(A_{\alpha} A_{\beta}\right)\right)^{2}\right\} .
\end{aligned}
$$

From equation (3.3), we know that

$$
\frac{1}{2} \Delta|\sigma|^{2}=\frac{1}{2} \sum_{\alpha=m+1}^{n+1} \Delta\left|A_{\alpha}\right|^{2}=\sum_{\alpha=m+1}^{n+1}\left\{\left|\nabla A_{\alpha}\right|^{2}+\left\langle\operatorname{trace} \nabla^{2} A_{\alpha}, A_{\alpha}\right\rangle\right\},
$$

and, in order to estimate this Laplacian, we first note that

$$
\begin{gathered}
\sum_{\alpha=m+1}^{n+1}\left(\operatorname{trace} A_{\alpha}\right)\left\langle A_{\alpha} T, T\right\rangle=m\langle\sigma(T, T), H\rangle, \\
\sum_{\alpha=m+1}^{n+1}\left(\operatorname{trace} A_{\alpha}\right)\langle H, N\rangle\left\langle E_{\alpha}, N\right\rangle=m\langle H, N\rangle^{2}, \\
\sum_{\alpha=m+1}^{n+1}\left(\operatorname{trace}\left(A_{N} A_{\alpha}\right)\right)\left\langle E_{\alpha}, N\right\rangle=\left|A_{N}\right|^{2}, \sum_{\alpha=m+1}^{n+1}\left(\operatorname{trace} A_{\alpha}\right)^{2}=m^{2}|H|^{2},
\end{gathered}
$$

and, since $s_{\alpha \beta}=-s_{\beta \alpha}$, that

$$
\sum_{\alpha, \beta=m+1}^{n+1}\left(\nabla_{E_{i}} s_{\alpha \beta}\right)\left(E_{i}\right) \operatorname{trace}\left(A_{\alpha} A_{\beta}\right)=0 .
$$

Next, we easily get

$$
\begin{aligned}
\left(\nabla^{\perp} \sigma\right)(X, Y, Z) & =\nabla_{X}^{\perp} \sigma(Y, Z)-\sigma\left(\nabla_{X} Y, Z\right)-\sigma\left(Y, \nabla_{X} Z\right) \\
& =\sum_{\alpha=m+1}^{n+1}\left\langle\left(\nabla_{X} A_{\alpha}\right) Y-\sum_{\beta=m+1}^{n+1} s_{\alpha \beta}(X) A_{\beta} Y, Z\right\rangle E_{\alpha}
\end{aligned}
$$


for all tangent vector fields $X, Y$ and $Z$, and then

$$
\begin{aligned}
& \left|\nabla^{\perp} \sigma\right|^{2}=\sum_{i, j, k=1}^{m}\left|\left(\nabla^{\perp} \sigma\right)\left(E_{i}, E_{j}, E_{k}\right)\right|^{2} \\
& =\sum_{\alpha=m+1}^{n+1} \sum_{i, j=1}^{m}\left\langle\left(\nabla_{E_{i}} A_{\alpha}\right) E_{j}-\sum_{\beta=m+1}^{n+1} s_{\alpha \beta}\left(E_{i}\right) A_{\beta} E_{j},\left(\nabla_{E_{i}} A_{\alpha}\right) E_{j}-\sum_{\gamma=m+1}^{n+1} s_{\alpha \gamma}\left(E_{i}\right) A_{\gamma} E_{j}\right\rangle,
\end{aligned}
$$

which means that

$$
\begin{aligned}
\left|\nabla^{\perp} \sigma\right|^{2}= & \sum_{\alpha=m+1}^{n+1}\left\{\left|\nabla A_{\alpha}\right|^{2}+\sum_{i=1}^{m}\left(\sum_{\beta=m+1}^{n+1} 2 s_{\alpha \beta}\left(E_{i}\right) \operatorname{trace}\left(\left(\nabla_{E_{i}} A_{\beta}\right) A_{\alpha}\right)\right.\right. \\
& \left.\left.-\sum_{\beta, \gamma=m+1}^{n+1} s_{\alpha \gamma}\left(E_{i}\right) s_{\gamma \beta}\left(E_{i}\right) \operatorname{trace}\left(A_{\alpha} A_{\beta}\right)\right)\right\} .
\end{aligned}
$$

Using (3.15) and (3.16), we can state the following proposition.

Proposition 3.1. Let $\Sigma^{m}$ be a pmc submanifold of $M^{n}(c) \times \mathbb{R}$, with mean curvature vector field $H$, shape operator $A$, and second fundamental form $\sigma$. Then we have

$$
\begin{aligned}
\frac{1}{2} \Delta|\sigma|^{2}= & \left|\nabla^{\perp} \sigma\right|^{2}+c\left\{\left(m-|T|^{2}\right)|\sigma|^{2}-2 m \sum_{\alpha=m+1}^{n+1}\left|A_{\alpha} T\right|^{2}\right. \\
& \left.+3 m\langle\sigma(T, T), H\rangle+m\left|A_{N}\right|^{2}-m^{2}\langle H, N\rangle^{2}-m^{2}|H|^{2}\right\} \\
& +\sum_{\alpha, \beta=m+1}^{n+1}\left\{\left(\operatorname{trace} A_{\beta}\right)\left(\operatorname{trace}\left(A_{\alpha}^{2} A_{\beta}\right)\right)+\operatorname{trace}\left[A_{\alpha}, A_{\beta}\right]^{2}-\left(\operatorname{trace}\left(A_{\alpha} A_{\beta}\right)\right)^{2}\right\},
\end{aligned}
$$

where $\left\{E_{\alpha}\right\}_{\alpha=m+1}^{n+1}$ is a local orthonormal frame field in the normal bundle.

Corollary 3.2. If $\Sigma^{m}$ is a minimal submanifold of $M^{n}(c) \times \mathbb{R}$, then we have

$$
\begin{aligned}
\frac{1}{2} \Delta|\sigma|^{2}= & \left|\nabla^{\perp} \sigma\right|^{2}+c\left\{\left(m-|T|^{2}\right)|\sigma|^{2}-2 m \sum_{\alpha=m+1}^{n+1}\left|A_{\alpha} T\right|^{2}+m\left|A_{N}\right|^{2}\right\} \\
& +\sum_{\alpha, \beta=m+1}^{n+1}\left\{\operatorname{trace}\left[A_{\alpha}, A_{\beta}\right]^{2}-\left(\operatorname{trace}\left(A_{\alpha} A_{\beta}\right)\right)^{2}\right\} .
\end{aligned}
$$

Now, let us consider a non-minimal pmc submanifold $\Sigma^{m}$ of $M^{n}(c) \times \mathbb{R}$. For any normal vector field $V$, define $\phi_{V}=A_{V}-\frac{\text { trace } A_{V}}{m} \mathrm{I}$, the traceless part of $A_{V}$. We shall also consider $\phi$ the traceless part of $\sigma$, given by

$$
\phi(X, Y)=\sigma(X, Y)-\langle X, Y\rangle H .
$$

It is easy to see that $\nabla^{\perp} \phi=\nabla^{\perp} \sigma,|\sigma|^{2}=|\phi|^{2}+m|H|^{2}$ and $\left|A_{V}\right|^{2}=\left|\phi_{V}\right|^{2}+$ (trace $\left.A_{V}\right)^{2} / m$. It is also easy to obtain, from the Ricci equation, that if a normal vector field $V$ is parallel in the normal bundle, then $\left[A_{V}, A_{U}\right]=0$ for all normal vector fields $U$. 
Let $\left\{E_{m+1}, \ldots, E_{n+1}\right\}$ be a local orthonormal frame field in the normal bundle such that $E_{m+1}=H /|H|$. Then, we obtain the following corollary directly from Proposition 3.1.

Corollary 3.3. If $\Sigma^{m}$ is a non-minimal pmc submanifold of $M^{n}(c) \times \mathbb{R}$, then we have

$$
\begin{aligned}
\frac{1}{2} \Delta|\phi|^{2}= & \left|\nabla^{\perp} \phi\right|^{2}+\left(c\left(m-|T|^{2}\right)+m|H|^{2}\right)|\phi|^{2}-2 c m \sum_{\alpha=m+1}^{n+1}\left|\phi_{\alpha} T\right|^{2} \\
& -c m\langle\phi(T, T), H\rangle+c m\left|\phi_{N}\right|^{2}+m|H| \sum_{\alpha=m+1}^{n+1} \operatorname{trace}\left(\phi_{\alpha}^{2} \phi_{m+1}\right) \\
& +\sum_{\alpha, \beta>m+1} \operatorname{trace}\left[\phi_{\alpha}, \phi_{\beta}\right]^{2}-\sum_{\alpha, \beta=m+1}^{n+1}\left(\operatorname{trace}\left(\phi_{\alpha} \phi_{\beta}\right)\right)^{2} .
\end{aligned}
$$

In the following, we shall compute the Laplacian of the squared norm of the tangent part $T$ of $\xi$.

As above, let us consider an orthonormal basis $\left\{e_{i}\right\}_{i=1}^{m}$ in $T_{p} \Sigma^{m}, p \in \Sigma^{m}$, and then extend $e_{i}$ to vector fields $E_{i}$ in a neighborhood of $p$ such that $\left\{E_{i}\right\}$ is a geodesic frame field around $p$. Then, at $p$, we have

$$
\frac{1}{2} \Delta|T|^{2}=\sum_{i=1}^{m}\left(\left\langle\nabla_{E_{i}} T, \nabla_{E_{i}} T\right\rangle+\left\langle\nabla_{E_{i}} \nabla_{E_{i}} T, T\right\rangle\right)=\left|A_{N}\right|^{2}+\sum_{i=1}^{m}\left\langle\nabla_{E_{i}} A_{N} E_{i}, T\right\rangle
$$

and, since $\nabla_{X} A_{N}$ is symmetric,

$$
\begin{aligned}
& \sum_{i=1}^{m}\left\langle\nabla_{E_{i}} A_{N} E_{i}, T\right\rangle=\sum_{i=1}^{m}\left\langle\left(\nabla_{E_{i}} A_{N}\right) E_{i}, T\right\rangle=\sum_{i=1}^{m}\left\langle\left(\nabla_{E_{i}} A_{N}\right) T, E_{i}\right\rangle \\
& =\sum_{i=1}^{m}\left\langle\nabla_{E_{i}} A_{N} T-A_{N} \nabla_{E_{i}} T, E_{i}\right\rangle=\sum_{i=1}^{m}\left\langle\nabla_{E_{i}} \nabla_{T} T-\nabla_{\nabla_{E_{i}} T} T, E_{i}\right\rangle \\
& =\sum_{i=1}^{m}\left\langle\nabla_{E_{i}} \nabla_{T} T+\nabla_{\left[T, E_{i}\right]} T, E_{i}\right\rangle=\sum_{i=1}^{m}\left(\left\langle\nabla_{T} \nabla_{E_{i}} T, E_{i}\right\rangle-\left\langle R\left(T, E_{i}\right) T, E_{i}\right\rangle\right) \\
& =\sum_{i=1}^{m}\left(\left\langle\nabla_{T} A_{N} E_{i}, E_{i}\right\rangle-\left\langle R\left(T, E_{i}\right) T, E_{i}\right\rangle\right)=T\left(\operatorname{trace} A_{N}\right)-\sum_{i=1}^{m}\left\langle R\left(T, E_{i}\right) T, E_{i}\right\rangle \\
& =m T(\langle H, N\rangle)-\sum_{i=1}^{m}\left\langle R\left(T, E_{i}\right) T, E_{i}\right\rangle=-m\langle\sigma(T, T), H\rangle-\sum_{i=1}^{m}\left\langle R\left(T, E_{i}\right) T, E_{i}\right\rangle
\end{aligned}
$$

where we used $\nabla_{X} T=A_{N} X$ and $\nabla_{X}^{\perp} N=-\sigma(X, T)$.

From the Gauss equation (2.2), it follows that

$$
\sum_{i=1}^{m}\left\langle R\left(T, E_{i}\right) T, E_{i}\right\rangle=c(1-m)|T|^{2}\left(1-|T|^{2}\right)+\sum_{\alpha=m+1}^{n+1}\left\{\left|A_{\alpha} T\right|^{2}-\left(\operatorname{trace} A_{\alpha}\right)\left\langle A_{\alpha} T, T\right\rangle\right\},
$$


and then we get

$$
\begin{aligned}
\frac{1}{2} \Delta|T|^{2}= & \left|A_{N}\right|^{2}-m\langle\sigma(T, T), H\rangle+c(m-1)|T|^{2}\left(1-|T|^{2}\right) \\
& -\sum_{\alpha=m+1}^{n+1}\left\{\left|A_{\alpha} T\right|^{2}-\left(\operatorname{trace} A_{\alpha}\right)\left\langle A_{\alpha} T, T\right\rangle\right\},
\end{aligned}
$$

where $\left\{E_{\alpha}\right\}_{\alpha=m+1}^{n+1}$ is a local orthonormal frame field in the normal bundle.

We conclude with the following proposition.

Proposition 3.4. Let $\Sigma^{m}$ be an $m$-dimensional pmc submanifold in $M^{n}(c) \times \mathbb{R}$, with shape operator $A$. Then we have

$$
\frac{1}{2} \Delta|T|^{2}=\left|A_{N}\right|^{2}+c(m-1)|T|^{2}\left(1-|T|^{2}\right)-\sum_{\alpha=m+1}^{n+1}\left|A_{\alpha} T\right|^{2} .
$$

\section{Some gap theorems for pmc submanifolds in $M^{n}(c) \times \mathbb{R}$}

In this section we shall present some applications of Propositions 3.1 and 3.4 in the study of pmc submanifolds. First we have the following result.

Proposition 4.1. Let $\Sigma^{m}$ be an immersed complete pmc submanifold in $M^{n}(c) \times \mathbb{R}$ with second fundamental form $\sigma$. If

$$
\sup _{\Sigma^{m}}\left\{|\sigma|^{2}+c(m-1)|T|^{2}\right\}<\max \{0, c(m-1)\}
$$

then either

1. $\Sigma^{m}$ lies in $M^{n}(c)$, if $c>0$; or

2. $\Sigma^{m}$ is a vertical cylinder $\pi^{-1}\left(\Sigma^{m-1}\right)$ over a pmc submanifold $\Sigma^{m-1}$ in the space form $M^{n}(c)$, if $c<0$.

Proof. Let us consider first the case when $c>0$. Then, from Proposition 3.4, using our hypothesis, we have that

$$
\begin{aligned}
\frac{1}{2} \Delta|T|^{2} & =\left|A_{N}\right|^{2}+c(m-1)|T|^{2}\left(1-|T|^{2}\right)-\sum_{\alpha=m+1}^{n+1}\left|A_{\alpha} T\right|^{2} \\
& \geq|T|^{2}\left(c(m-1)\left(1-|T|^{2}\right)-|\sigma|^{2}\right) \geq 0 .
\end{aligned}
$$

Next, let us consider a local orthonormal frame field $\left\{E_{i}\right\}_{i=1}^{m}$ on $\Sigma^{m}, X$ a unit tangent vector field, and an orthonormal frame field $\left\{E_{\alpha}\right\}_{\alpha=m+1}^{n+1}$ in the normal bundle. From equation (2.2), we get the expression of the Ricci curvature of our 
submanifold

$$
\begin{aligned}
\operatorname{Ric} X= & \sum_{i=1}^{m}\left\langle R\left(E_{i}, X\right) X, E_{i}\right\rangle \\
= & \sum_{i=1}^{m}\left\{c \left(|X|^{2}-\left\langle X, E_{i}\right\rangle^{2}-\langle X, T\rangle^{2}+2\langle X, T\rangle\left\langle T, E_{i}\right\rangle\left\langle X, E_{i}\right\rangle\right.\right. \\
& \left.\left.-\left\langle T, E_{i}\right\rangle^{2}|X|^{2}\right)+\sum_{\alpha=m+1}^{n+1}\left(\left\langle A_{\alpha} E_{i}, E_{i}\right\rangle\left\langle A_{\alpha} X, X\right\rangle-\left\langle A_{\alpha} X, E_{i}\right\rangle^{2}\right)\right\} \\
= & c\left(m-1-|T|^{2}-(m-2)\langle X, T\rangle^{2}\right)+m\left\langle A_{H} X, X\right\rangle-\sum_{\alpha=m+1}^{n+1}\left|A_{\alpha} X\right|^{2} .
\end{aligned}
$$

It follows that

$$
\operatorname{Ric} X \geq c(m-1)\left(1-|T|^{2}\right)-m\left|A_{H} X\right|-\sum_{\alpha=m+1}^{n+1}\left|A_{\alpha}\right|^{2} \geq-m\left|A_{H}\right|-|\sigma|^{2} .
$$

Since by hypothesis $|\sigma|$ is bounded, we can see that the Ricci curvature is bounded from below, and then the Omori-Yau maximum principle holds on $\Sigma^{m}$.

Therefore, we can use Theorem 2.6 with $u=|T|^{2}$. It follows that there exists a sequence of points $\left\{p_{k}\right\}_{k \in \mathbb{N}} \subset \Sigma^{m}$ satisfying

$$
\lim _{k \rightarrow \infty}|T|^{2}\left(p_{k}\right)=\sup _{\Sigma^{m}}|T|^{2} \quad \text { and } \quad \Delta|T|^{2}\left(p_{k}\right)<\frac{1}{k}
$$

Since $\sup _{\Sigma^{m}}\left\{|\sigma|^{2}+c(m-1)|T|^{2}\right\}<c(m-1)$, it follows that $0=\lim _{k \rightarrow \infty}|T|^{2}\left(p_{k}\right)=$ $\sup _{\Sigma^{m}}|T|^{2}$, which means that $T=0$, i.e., $\Sigma^{m}$ lies in $M^{n}(c)$.

When $c<0$, we come to the conclusion in the same way as above, using that

$$
\begin{aligned}
\frac{1}{2} \Delta|N|^{2} & =-\frac{1}{2} \Delta|T|^{2}=-\left|A_{N}\right|^{2}-c(m-1)|T|^{2}\left(1-|T|^{2}\right)+\sum_{\alpha=m+1}^{n+1}\left|A_{\alpha} T\right|^{2} \\
& \geq|N|^{2}\left(-|\sigma|^{2}-c(m-1)|T|^{2}\right) \geq 0,
\end{aligned}
$$

and that

$$
\text { Ric } X \geq c(m-1)-m\left|A_{H}\right|-|\sigma|^{2},
$$

and then applying Theorem 2.6 to function $u=|N|^{2}$.

For minimal submanifolds in $M^{n}(c) \times \mathbb{R}$, with $c>0$, we have the following:

Proposition 4.2. Let $\Sigma^{m}$ be a complete minimal submanifold in $M^{n}(c) \times \mathbb{R}$, with $c>0$. If

$$
\sup _{\Sigma^{m}}\left\{3|\sigma|^{2}+2 c(2 m+1)|T|^{2}\right\}<2 c m,
$$

then $\Sigma^{m}$ is a totally geodesic submanifold in $M^{n}(c)$.

Proof. From Corollary 3.2, since Schwarz inequality implies $\left|A_{\alpha} T\right|^{2} \leq|T|^{2}\left|A_{\alpha}\right|^{2}$, using $\left|A_{N}\right|^{2} \geq 0$ and Lemma 2.5, we obtain

$$
\Delta|\sigma|^{2} \geq-\left(3|\sigma|^{2}+2 c\left((2 m+1)|T|^{2}-m\right)\right)|\sigma|^{2} \geq 0
$$


As we have seen, since $|\sigma|$ is bounded, the Ricci curvature of $\Sigma^{m}$ is bounded from below, and then we can apply the Omori-Yau maximum principle to the function $u=|\sigma|^{2}$. One obtains that there exists a sequence of points $\left\{p_{k}\right\}_{k \in \mathbb{N}} \subset \Sigma^{m}$ satisfying

$$
\lim _{k \rightarrow \infty}|\sigma|^{2}\left(p_{k}\right)=\sup _{\Sigma^{m}}|\sigma|^{2} \quad \text { and } \quad \Delta|\sigma|^{2}\left(p_{k}\right)<\frac{1}{k}
$$

from where it follows that $0=\lim _{k \rightarrow \infty}|\sigma|^{2}\left(p_{k}\right)=\sup _{\Sigma^{m}}|\sigma|^{2}$, which means that $\sigma=0$. Moreover, $A_{N}=0$ and then the hypotheses imply that $|T|^{2}=$ constant $<1$. From Proposition 3.4, it follows that $T=0$, which means that our submanifold is totally geodesic in $M^{n}(c)$.

Before stating our first main result, we shall prove the following lemma, which will then be used in its proof.

Lemma 4.3. Let $\Sigma^{m}$ be an immersed non-minimal pmc submanifold in $M^{n}(c) \times \mathbb{R}$ with mean curvature vector field $H$. Then we have

$$
\Delta\langle H, N\rangle=-c(m-1)|T|^{2}\langle H, N\rangle-\operatorname{trace}\left(A_{H} A_{N}\right) .
$$

Proof. Let $\left\{E_{i}\right\}_{i=1}^{m}$ be a geodesic frame field around a point $p \in \Sigma^{m}$. Then, since $H$ is parallel and $\nabla_{X}^{\perp} N=-\sigma(X, T)$, we have, at $p$,

$$
\Delta\langle H, N\rangle=\sum_{i=1}^{m} E_{i}\left(E_{i}(\langle H, N\rangle)\right)=-\sum_{i=1}^{m} E_{i}\left(\left\langle A_{H} T, E_{i}\right\rangle\right) .
$$

Using the facts that $\nabla_{X} A_{H}$ is symmetric and that $\nabla_{X} T=A_{N} X$, and also equation (3.7), we get

$$
\begin{aligned}
\Delta\langle H, N\rangle & =-\sum_{i=1}^{m} E_{i}\left(\left\langle A_{H} T, E_{i}\right\rangle\right)=-\sum_{i=1}^{m}\left\langle\nabla_{E_{i}} A_{H} T, E_{i}\right\rangle \\
& =-\sum_{i=1}^{m}\left(\left\langle\left(\nabla_{E_{i}} A_{H}\right) T, E_{i}\right\rangle+\left\langle A_{H} \nabla_{E_{i}} T, E_{i}\right\rangle\right) \\
& =-\sum_{i=1}^{m}\left\langle\left(\nabla_{E_{i}} A_{H}\right) E_{i}, T\right\rangle-\operatorname{trace}\left(A_{H} A_{N}\right) \\
& =-c(m-1)|T|^{2}\langle H, N\rangle-\operatorname{trace}\left(A_{H} A_{N}\right) .
\end{aligned}
$$

Our main results are similar to those obtained in [5], [8] for the pmc submanifolds of a sphere and Euclidean space, and as in the above cited papers, their proofs rely on the use of the formulas obtained in Section 3 and Lemmas 2.4 and 2.5.

Theorem 4.4. Let $\Sigma^{m}$ be a complete non-minimal pmc submanifold in $M^{n}(c) \times \mathbb{R}$, $n>m \geq 3, c>0$, with mean curvature vector field $H$ and second fundamental form $\sigma$. If the angle between $H$ and $\xi$ is constant and

$$
|\sigma|^{2}+\frac{2 c(2 m+1)}{m}|T|^{2} \leq 2 c+\frac{m^{2}}{m-1}|H|^{2},
$$

then $\Sigma^{m}$ is a totally umbilical cmc hypersurface in $M^{m+1}(c)$. 
Proof. We shall prove first that $\Sigma^{m}$ actually lies in a space form $M^{m+1}(c)$, and, in order to do that, we will show that, if $\left\{E_{m+1}, \ldots, E_{n+1}\right\}$ is a local orthonormal frame field in the normal bundle such that $E_{m+1}=H /|H|$, then $A_{\alpha}=0$ for all $\alpha>m+1$.

Let us recall now the following formula proved in [12], which can be also obtained as a particular case of the computation in Section 3, tacking into account that, since $E_{m+1}$ is parallel, we have $\left[A_{m+1}, A_{\alpha}\right]=0$ for all $\alpha \geq m+1$ :

$$
\begin{aligned}
\frac{1}{2} \Delta\left|A_{m+1}\right|^{2}= & \left|\nabla A_{m+1}\right|^{2}+c\left\{\left(m-|T|^{2}\right)\left|A_{m+1}\right|^{2}\right. \\
& -2 m\left|A_{m+1} T\right|^{2}+3 m\langle\sigma(T, T), H\rangle \\
& \left.+m\left(\operatorname{trace}\left(A_{N} A_{m+1}\right)\right)\left\langle E_{m+1}, N\right\rangle-m^{2}\langle H, N\rangle^{2}-m^{2}|H|^{2}\right\} \\
& +\sum_{\alpha=m+1}^{n+1}\left\{\left(\operatorname{trace} A_{\alpha}\right)\left(\operatorname{trace}\left(A_{m+1}^{2} A_{\alpha}\right)\right)-\left(\operatorname{trace}\left(A_{m+1} A_{\alpha}\right)\right)^{2}\right\} .
\end{aligned}
$$

Next, we define the function $|\mathcal{A}|^{2}$ on $\Sigma^{m}$ by $|\mathcal{A}|^{2}=\sum_{\alpha>m+1}\left|A_{\alpha}\right|^{2}$, and, using (4.2), we obtain, from Proposition 3.1, that

$$
\begin{aligned}
\frac{1}{2} \Delta|\mathcal{A}|^{2}= & \sum_{\alpha>m+1}\left|\nabla^{*} A_{\alpha}\right|^{2}+c\left\{\left(m-|T|^{2}\right)|\mathcal{A}|^{2}-2 m \sum_{\alpha>m+1}\left|A_{\alpha} T\right|^{2}\right. \\
& \left.+m\left|A_{N}\right|^{2}-m\left(\operatorname{trace}\left(A_{N} A_{m+1}\right)\right)\left\langle E_{m+1}, N\right\rangle\right\} \\
& +\sum_{\alpha>m+1}\left\{\left(\operatorname{trace} A_{m+1}\right)\left(\operatorname{trace}\left(A_{\alpha}^{2} A_{m+1}\right)\right)-\left(\operatorname{trace}\left(A_{\alpha} A_{m+1}\right)\right)^{2}\right\} \\
& +\sum_{\alpha, \beta>m+1}\left\{\operatorname{trace}\left[A_{\alpha}, A_{\beta}\right]^{2}-\left(\operatorname{trace}\left(A_{\alpha} A_{\beta}\right)\right)^{2}\right\}
\end{aligned}
$$

where $\nabla^{*}$ is the sum of the tangent and normal connections and

$$
\nabla_{X}^{*} A_{\alpha}=\nabla_{X} A_{\alpha}-\sum_{\beta>m+1} s_{\alpha \beta}(X) A_{\beta} .
$$

The Schwarz inequality implies that

$$
-\sum_{\alpha>m+1}\left|A_{\alpha} T\right|^{2} \geq-|T|^{2} \sum_{\alpha>m+1}\left|A_{\alpha}\right|^{2}=-|T|^{2}|\mathcal{A}|^{2} .
$$

From Lemma 4.3, since $\langle H, N\rangle=$ constant, we have

(4.5) $\left|A_{N}\right|^{2}-\left(\operatorname{trace}\left(A_{N} A_{m+1}\right)\right)\left\langle E_{m+1}, N\right\rangle=\left|A_{N}\right|^{2}+c(m-1)|T|^{2}\left\langle E_{m+1}, N\right\rangle^{2} \geq 0$.

Since trace $\left[A_{\alpha}, A_{\beta}\right]^{2}=-N\left(A_{\alpha} A_{\beta}-A_{\beta} A_{\alpha}\right)$, using Lemma 2.5, we get

$$
\sum_{\alpha, \beta>m+1}\left\{\operatorname{trace}\left[A_{\alpha}, A_{\beta}\right]^{2}-\left(\operatorname{trace}\left(A_{\alpha} A_{\beta}\right)\right)^{2}\right\} \geq-\frac{3}{2}\left(\sum_{\alpha>m+1}\left|A_{\alpha}\right|^{2}\right)^{2}=-\frac{3}{2}|\mathcal{A}|^{4}
$$

Next, we shall evaluate the term

$$
\sum_{\alpha>m+1}\left\{\left(\operatorname{trace} A_{m+1}\right)\left(\operatorname{trace}\left(A_{\alpha}^{2} A_{m+1}\right)\right)-\left(\operatorname{trace}\left(A_{\alpha} A_{m+1}\right)\right)^{2}\right\}
$$


in (4.3). In order to do so, we note first that, since $\left[A_{m+1}, A_{\alpha}\right]=0$, the matrices $A_{m+1}$ and $A_{\alpha}$ can be diagonalized simultaneously, for each $\alpha>m+1$. Let $\lambda_{i}$ and $\lambda_{i}^{\alpha}, i=1, \ldots, m$, be the eigenvalues of $A_{m+1}$ and $A_{\alpha}$, respectively. Then, for each $\alpha>m+1$, we have

$$
\begin{aligned}
& \left(\operatorname{trace} A_{m+1}\right)\left(\operatorname{trace}\left(A_{\alpha}^{2} A_{m+1}\right)\right)-\left(\operatorname{trace}\left(A_{\alpha} A_{m+1}\right)\right)^{2} \\
& =\left(\sum_{i=1}^{m} \lambda_{i}\right)\left(\sum_{j=1}^{m} \lambda_{j}\left(\lambda_{j}^{\alpha}\right)^{2}\right)-\left(\sum_{i=1}^{m} \lambda_{i} \lambda_{i}^{\alpha}\right)\left(\sum_{j=1}^{m} \lambda_{j} \lambda_{j}^{\alpha}\right)=\frac{1}{2} \sum_{i, j=1}^{m} \lambda_{i} \lambda_{j}\left(\lambda_{i}^{\alpha}-\lambda_{j}^{\alpha}\right)^{2} .
\end{aligned}
$$

Our hypothesis (4.1) can be written as

$$
(m|H|)^{2} \geq(m-1)\left|A_{m+1}\right|^{2}+(m-1)\left(|\mathcal{A}|^{2}+\frac{2 c(2 m+1)}{m}|T|^{2}-2 c\right)
$$

which means that

$$
\left(\sum_{i=1}^{m} \lambda_{i}\right)^{2} \geq(m-1) \sum_{i=1}^{m}\left(\lambda_{i}\right)^{2}+(m-1)\left(|\mathcal{A}|^{2}+\frac{2 c(2 m+1)}{m}|T|^{2}-2 c\right) .
$$

Thus, from Lemma 2.4, it follows that

$$
\lambda_{i} \lambda_{j} \geq \frac{1}{2}|\mathcal{A}|^{2}+\frac{c(2 m+1)}{m}|T|^{2}-c,
$$

for $i \neq j$, and then

$$
\begin{aligned}
\frac{1}{2} \sum_{i, j=1}^{m} \lambda_{i} \lambda_{j}( & \left.\lambda_{i}^{\alpha}-\lambda_{j}^{\alpha}\right)^{2} \geq \frac{1}{2}\left(\frac{1}{2}|\mathcal{A}|^{2}+\frac{c(2 m+1)}{m}|T|^{2}-c\right) \sum_{i, j=1}^{m}\left(\lambda_{i}^{\alpha}-\lambda_{j}^{\alpha}\right)^{2} \\
= & \left(\frac{1}{2}|\mathcal{A}|^{2}+\frac{c(2 m+1)}{m}|T|^{2}-c\right) \sum_{i, j=1}^{m}\left(\left(\lambda_{i}^{\alpha}\right)^{2}-\lambda_{i}^{\alpha} \lambda_{j}^{\alpha}\right) \\
= & \left(\frac{m}{2}|\mathcal{A}|^{2}+c(2 m+1)|T|^{2}-c m\right)\left|A_{\alpha}\right|^{2} \\
& -\left(\frac{1}{2}|\mathcal{A}|^{2}+\frac{c(2 m+1)}{m}|T|^{2}-c\right)\left(\sum_{i=1}^{m} \lambda_{i}^{\alpha}\right)^{2} \\
= & \left(\frac{m}{2}|\mathcal{A}|^{2}+c(2 m+1)|T|^{2}-c m\right)\left|A_{\alpha}\right|^{2} .
\end{aligned}
$$

Substituting this in (4.7), we get

$$
\begin{aligned}
\sum_{\alpha>m+1}\left\{\left(\operatorname{trace} A_{m+1}\right)\left(\operatorname{trace}\left(A_{\alpha}^{2} A_{m+1}\right)\right)-\left(\operatorname{trace}\left(A_{\alpha} A_{m+1}\right)\right)^{2}\right\} & \\
& \geq\left(\frac{m}{2}|\mathcal{A}|^{2}+c(2 m+1)|T|^{2}-c m\right)|\mathcal{A}|^{2} .
\end{aligned}
$$

Now, from (4.3), (4.4), (4.5), (4.6) and (4.11), one obtains

$$
\frac{1}{2} \Delta|\mathcal{A}|^{2} \geq \frac{m-3}{2}|\mathcal{A}|^{4} .
$$


As we have seen in Proposition 4.1, the fact that $|\sigma|$ is bounded implies that the Ricci curvature of $\Sigma^{m}$ is bounded from below. Therefore we can apply Theorem 2.6 to the function $u=|\mathcal{A}|^{2}$, and we get that there exists a sequence of points $\left\{p_{k}\right\}_{k \in \mathbb{N}} \subset \Sigma^{m}$ satisfying

$$
\lim _{k \rightarrow \infty}|\mathcal{A}|^{2}\left(p_{k}\right)=\sup _{\Sigma^{m}}|\mathcal{A}|^{2} \quad \text { and } \quad \Delta|\mathcal{A}|^{2}\left(p_{k}\right)<\frac{1}{k} .
$$

From the inequality (4.12) it follows that

$$
0=\lim _{k \rightarrow \infty} \Delta|\mathcal{A}|^{2}\left(p_{k}\right) \geq(m-3) \sup _{\Sigma^{m}}|\mathcal{A}|^{2} \geq 0,
$$

i.e., $(m-3) \sup _{\Sigma^{m}}|\mathcal{A}|^{2}=0$. Therefore, we get that $m=3$ or $|\mathcal{A}|^{2}=0$.

Next, we shall split our study in two cases as $m \geq 4$ or $m=3$.

Case I: $m \geq 4$. In this case, we have $|\mathcal{A}|^{2}=0$, and then $A_{\alpha}=0$ for all $\alpha>m+1$. Moreover, all the inequalities (4.4), (4.5), (4.6) and (4.11) become equalities. Since $A_{N}=0$, we get that $|T|^{2}$ is constant and that $\langle H, N\rangle=0$. We also have

$$
0=X(\langle H, N\rangle)=\left\langle H, \nabla_{X}^{\perp} N\right\rangle=-|H|\left\langle E_{m+1}, \sigma(T, X)\right\rangle=-|H|\left\langle A_{m+1} T, X\right\rangle,
$$

for any tangent vector field $X$. Therefore, from Proposition 3.4, it follows that

$$
0=c(m-1)|T|^{2}\left(1-|T|^{2}\right),
$$

i.e., either $T=0$ or $T= \pm \xi$.

If $T= \pm \xi$, then $\Sigma^{m}$ is a vertical cylinder $\pi^{-1}\left(\Sigma^{m-1}\right)$ over a pmc submanifold $\Sigma^{m-1}$ in $M^{n}(c)$ with second fundamental form $\sigma_{0}$, satisfying $\left|\sigma_{0}\right|=|\sigma|$, and mean curvature vector field $H_{0}=\frac{m}{m-1} H$. Then, condition (4.1) can be rewritten as

$$
\left|\sigma_{0}\right|^{2} \leq(m-1)\left|H_{0}\right|^{2}-\frac{2 c(m+1)}{m}<(m-1)\left|H_{0}\right|^{2},
$$

which is a contradiction, since the squared norm of the traceless part $\phi_{0}$ of $\sigma_{0}$ satisfies

$$
0 \leq\left|\phi_{0}\right|^{2}=\left|\sigma_{0}\right|^{2}-(m-1)\left|H_{0}\right|^{2} .
$$

Hence, we have $T=0$, i.e., $\xi$ is normal to $\Sigma^{m}$. Since $A_{\alpha}=0$ for all $\alpha>m+1$, it follows that the subbundle $L=\operatorname{span}\{\sigma\}=\operatorname{span}\{H\}$ of the normal bundle is parallel, i.e., $\nabla^{\perp} V \in L$ for all $V \in L$. Now, one can see that $T \Sigma^{m} \oplus L$ is parallel, orthogonal to $\xi$, and invariant by the curvature tensor $\bar{R}$. Using Theorem 2 in [11], all these lead to the conclusion that $\Sigma^{m}$ lies in an $m+1$-dimensional totally geodesic submanifold of $M^{n}(c) \times \mathbb{R}$, which is also orthogonal to $\xi$, i.e., $\Sigma^{m}$ is a cmc hypersurface in $M^{m+1}(c)$.

Case II: $m=3$. We shall prove that $|\mathcal{A}|^{2}=0$ in this situation too, which means, as we have seen above, that $\Sigma^{3}$ is a cmc hypersurface in $M^{4}(c)$.

Our hypothesis (4.1) implies that the sequence $\left\{\sigma_{i j}^{\alpha}\left(p_{k}\right)\right\}_{k \in \mathbb{N}}$, where

$$
\sigma_{i j}^{\alpha}=\left\langle\sigma\left(E_{i}, E_{j}\right), E_{\alpha}\right\rangle,
$$


is bounded for all $i, j$ and $\alpha$. We also know that the sequence $\left\{|T|^{2}\left(p_{k}\right)\right\}_{k \in \mathbb{N}}$ is bounded. Therefore, there exists a subsequence $\left\{p_{k_{r}}\right\}_{k_{r} \in \mathbb{N}}$ of $\left\{p_{k}\right\}_{k \in \mathbb{N}}$ such that the following limits exist:

$$
\bar{\sigma}_{i j}^{\alpha}=\lim _{k_{r} \rightarrow \infty} \sigma_{i j}^{\alpha}\left(p_{k_{r}}\right)<\infty \text { and }|\bar{T}|^{2}=\lim _{k_{r} \rightarrow \infty}|T|^{2}\left(p_{k_{r}}\right)<\infty,
$$

and we denote by

$$
\bar{A}_{\alpha}=\lim _{k_{r} \rightarrow \infty} A_{\alpha}\left(p_{k_{r}}\right)
$$

the matrix with entries $\bar{\sigma}_{i j}^{\alpha}$.

From $\lim _{k_{r} \rightarrow \infty} \Delta|\mathcal{A}|^{2}\left(p_{k_{r}}\right)=0$, it follows that, when we take the limit after $k_{r} \rightarrow \infty$, all the inequalities (4.4), (4.5), (4.6), and (4.11) become equalities. Then, from (4.6) and (4.11) we obtain

$$
\sum_{\alpha, \beta>4}\left\{\operatorname{trace}\left[\bar{A}_{\alpha}, \bar{A}_{\beta}\right]^{2}-\left(\operatorname{trace}\left(\bar{A}_{\alpha} \bar{A}_{\beta}\right)\right)^{2}\right\}=-\frac{3}{2}\left(\sum_{\alpha>4}\left|\bar{A}_{\alpha}\right|^{2}\right)^{2}=-\frac{3}{2}\left(\sup _{\Sigma^{3}}|\mathcal{A}|^{2}\right)^{2}
$$

and

$$
\begin{aligned}
& \sum_{\alpha>4}\left\{\left(\operatorname{trace} \bar{A}_{4}\right)\left(\operatorname{trace}\left(\bar{A}_{\alpha}^{2} \bar{A}_{4}\right)\right)-\left(\operatorname{trace}\left(\bar{A}_{\alpha} \bar{A}_{4}\right)\right)^{2}\right\} \\
& =\left(\frac{3}{2} \sum_{\alpha>4}\left|\bar{A}_{\alpha}\right|^{2}+7 c|\bar{T}|^{2}-3 c\right) \sum_{\alpha>4}\left|\bar{A}_{\alpha}\right|^{2}=\left(\frac{3}{2} \sup _{\Sigma^{3}}|\mathcal{A}|^{2}+7 c|\bar{T}|^{2}-3 c\right) \sup _{\Sigma^{3}}|\mathcal{A}|^{2},
\end{aligned}
$$

respectively. From (4.13) and Lemma 2.5, it follows that either

1. $\bar{A}_{5}=\ldots=\bar{A}_{n+1}=0$; or

2. only two matrices $\bar{A}_{\alpha_{0}}$ and $\bar{A}_{\beta_{0}}$ are different from the null $m \times m$ matrix, $\left|\bar{A}_{\alpha_{0}}\right|^{2}=\left|\bar{A}_{\beta_{0}}\right|^{2}=L$, and there exists an orthogonal matrix $T$ such that

$$
T^{t} \bar{A}_{\alpha_{0}} T=\sqrt{\frac{L}{2}}\left(\begin{array}{ccc}
1 & 0 & 0 \\
0 & -1 & 0 \\
0 & 0 & 0
\end{array}\right), \quad T^{t} \bar{A}_{\beta_{0}} T=\sqrt{\frac{L}{2}}\left(\begin{array}{ccc}
0 & 1 & 0 \\
1 & 0 & 0 \\
0 & 0 & 0
\end{array}\right) .
$$

In the first case, one obtains

$$
0=\sum_{\alpha>4}\left|\bar{A}_{\alpha}\right|^{2}=\sup _{\Sigma^{3}}|\mathcal{A}|^{2}
$$

which means that $|\mathcal{A}|^{2}=0$ or, equivalently, that $A_{\alpha}=0$ for all $\alpha>4$.

In the following, we shall assume that the second case occurs, and we will come to a contradiction.

Restricting (4.10) to the sequence of points $\left\{p_{k_{r}}\right\}_{k_{r} \in \mathbb{N}}$ and then taking the limit, we get, also using (4.14), that

$$
\sum_{i, j=1}^{3} \bar{\lambda}_{i} \bar{\lambda}_{j}\left(\bar{\lambda}_{i}^{\alpha}-\bar{\lambda}_{j}^{\alpha}\right)^{2}=\left(\frac{1}{2} \sup _{\Sigma^{3}}|\mathcal{A}|^{2}+\frac{7 c}{3}|\bar{T}|^{2}-c\right) \sum_{i, j=1}^{3}\left(\bar{\lambda}_{i}^{\alpha}-\bar{\lambda}_{j}^{\alpha}\right)^{2},
$$

where $\bar{\lambda}_{i}=\lim _{k_{r} \rightarrow \infty} \lambda_{i}$ and $\bar{\lambda}_{i}^{\alpha}=\lim _{k_{r} \rightarrow \infty} \lambda_{i}^{\alpha}$. 
From (4.15) we have $\bar{\lambda}_{i}^{\alpha} \neq \bar{\lambda}_{j}^{\alpha}$ for $i \neq j$, and then, from (4.9), one obtains

$$
\bar{\lambda}_{i} \bar{\lambda}_{j}=\frac{1}{2} \sup _{\Sigma^{3}}|\mathcal{A}|^{2}+\frac{7 c}{3}|\bar{T}|^{2}-c \quad \text { for } \quad i \neq j .
$$

Now, on the one hand, taking the limit in (4.8) and applying Lemma 2.4, we get

$$
\left(\sum_{i=1}^{3} \bar{\lambda}_{i}\right)^{2}=2 \sum_{i=1}^{3}\left(\bar{\lambda}_{i}\right)^{2}+2\left(\sup _{\Sigma^{3}}|\mathcal{A}|^{2}+\frac{14 c}{3}|\bar{T}|^{2}-2 c\right),
$$

or, equivalently,

$$
\frac{3}{2}|H|^{2}=\left|\bar{\phi}_{4}\right|^{2}+\sup _{\Sigma^{3}}|\mathcal{A}|^{2}+\frac{14 c}{3}|\bar{T}|^{2}-2 c,
$$

where $\phi_{4}=A_{4}-|H| \mathrm{I}$ is the traceless part of $A_{4}$ and $\bar{\phi}_{4}=\lim _{k_{r} \rightarrow \infty} \phi_{4}\left(p_{k_{r}}\right)$.

On the other hand, we have

$$
\sum_{i \neq j} \lambda_{i} \lambda_{j}=\left(\sum_{i=1}^{3} \lambda_{i}\right)^{2}-\sum_{i=1}^{3}\left(\lambda_{i}\right)^{2}=9|H|^{2}-\left(\left|\phi_{4}\right|^{2}+3|H|^{2}\right)=6|H|^{2}-\left|\phi_{4}\right|^{2},
$$

which, taking the limit and using (4.16), gives

$$
\left|\bar{\phi}_{4}\right|^{2}=6|H|^{2}-3 \sup _{\Sigma^{3}}|\mathcal{A}|^{2}-14 c|\bar{T}|^{2}+6 c .
$$

Summarizing, from (4.17) and (4.18) one obtains

$$
\left|\bar{\phi}_{4}\right|^{2}=-\frac{3}{4}|H|^{2}
$$

which is a contradiction and, therefore, this case cannot occur.

We have just proved that our submanifold $\Sigma^{m}$ actually is a cmc hypersurface in $M^{m+1}(c)$ for any $m \geq 3$.

Now, from (4.1), it is easy to see that

$$
|\phi|^{2} \leq 2 c+\frac{m}{m-1}|H|^{2}<r^{2},
$$

where $\phi$ is the traceless part of $\sigma$ and $r$ is the positive root of the polynomial

$$
P(t)=t^{2}+\frac{m(m-2)}{\sqrt{m(m-1)}}|H| t-m\left(c+|H|^{2}\right) .
$$

We then use Theorem 1.5 in [2] (see also [17]) to conclude that $\phi=0$, i.e., $\Sigma^{m}$ is a totally umbilical cmc hypersurface in $M^{m+1}(c)$.

Theorem 4.5. Let $\Sigma^{m}$ be a complete non-minimal pmc submanifold in $M^{n}(c) \times \mathbb{R}$, $n>m \geq 3, c<0$, with mean curvature vector field $H$ and second fundamental form $\sigma$. If $H$ is orthogonal to $\xi$ and

$$
|\sigma|^{2}+\frac{2 c(m+1)}{m}|T|^{2} \leq 4 c+\frac{m^{2}}{m-1}|H|^{2},
$$

then $\Sigma^{m}$ is a totally umbilical cmc hypersurface in $M^{m+1}(c)$. 
Proof. Let us consider a local orthonormal frame field $\left\{E_{m+1}, \ldots, E_{n+1}\right\}$ in the normal bundle such that $E_{m+1}=H /|H|$. Then, since $H \perp \xi$, we have

$$
A_{N}=\sum_{\alpha>m+1}\left\langle N, E_{\alpha}\right\rangle A_{\alpha}
$$

and, therefore, from the Schwarz inequality, one obtains

$$
\begin{aligned}
\left|A_{N}\right|^{2} & =\left|\sum_{\alpha>m+1}\left\langle N, E_{\alpha}\right\rangle A_{\alpha}\right|^{2} \leq\left(\sum_{\alpha>m+1}\left|\left\langle N, E_{\alpha}\right\rangle\right|\left|A_{\alpha}\right|\right)^{2} \\
& \leq\left(\sum_{\alpha>m+1}\left|\left\langle N, E_{\alpha}\right\rangle\right|^{2}\right)\left(\sum_{\alpha>m+1}\left|A_{\alpha}\right|^{2}\right) \leq|N|^{2}|\mathcal{A}|^{2}=\left(1-|T|^{2}\right)|\mathcal{A}|^{2},
\end{aligned}
$$

where $|\mathcal{A}|^{2}=\sum_{\alpha>m+1}\left|A_{\alpha}\right|^{2}$. Then, from (4.3), it follows that

$$
\begin{aligned}
\frac{1}{2} \Delta|\mathcal{A}|^{2} \geq & c\left(2 m-(m+1)|T|^{2}\right)|\mathcal{A}|^{2} \\
& +\sum_{\alpha>m+1}\left\{\left(\operatorname{trace} A_{m+1}\right)\left(\operatorname{trace}\left(A_{\alpha}^{2} A_{m+1}\right)\right)-\left(\operatorname{trace}\left(A_{\alpha} A_{m+1}\right)\right)^{2}\right\} \\
& +\sum_{\alpha, \beta>m+1}\left\{\operatorname{trace}\left[A_{\alpha}, A_{\beta}\right]^{2}-\left(\operatorname{trace}\left(A_{\alpha} A_{\beta}\right)\right)^{2}\right\},
\end{aligned}
$$

where we also used the fact that $-c \sum_{\alpha>m+1}\left|A_{\alpha} T\right|^{2} \geq 0$.

Next, in the same way as in the proof of Theorem 4.4, we get

$$
\sum_{\alpha, \beta>m+1}\left\{\operatorname{trace}\left[A_{\alpha}, A_{\beta}\right]^{2}-\left(\operatorname{trace}\left(A_{\alpha} A_{\beta}\right)\right)^{2}\right\} \geq-\frac{3}{2}|\mathcal{A}|^{4}
$$

and, using (4.19),

$$
\begin{aligned}
\sum_{\alpha>m+1}\left\{\left(\operatorname{trace} A_{m+1}\right)\left(\operatorname{trace}\left(A_{\alpha}^{2} A_{m+1}\right)\right)-\left(\operatorname{trace}\left(A_{\alpha} A_{m+1}\right)\right)^{2}\right\} & \\
& \geq\left(\frac{m}{2}|\mathcal{A}|^{2}+c(m+1)|T|^{2}-2 c m\right)|\mathcal{A}|^{2} .
\end{aligned}
$$

Substituting this in (4.20), we obtain that

$$
\frac{1}{2} \Delta|\mathcal{A}|^{2} \geq \frac{m-3}{2}|\mathcal{A}|^{4}
$$

which, as in the proof of Theorem 4.4, implies that $|\mathcal{A}|^{2}=0$, and, therefore, $A_{\alpha}=0$ for all $\alpha>m+1$.

On the other hand, since $H \perp \xi$ implies that $A_{m+1} T=0$, and $A_{N}=0$ implies that $|T|=$ constant, from Proposition 3.4, we can see that

$$
0=c(m-1)|T|^{2}\left(1-|T|^{2}\right),
$$

which means that either $T=0$ or $T= \pm \xi$. If $T= \pm \xi$, then $\Sigma^{m}$ is a vertical cylinder $\pi^{-1}\left(\Sigma^{m-1}\right)$ over a pmc submanifold $\Sigma^{m-1}$ in $M^{n}(c)$, with second fundamental form $\sigma_{0}$, satisfying $\left|\sigma_{0}\right|=|\sigma|$, and mean curvature vector field $H_{0}=\frac{m}{m-1} H$. 
Then, from (4.19), it follows that

$$
\left|\sigma_{0}\right|^{2} \leq(m-1)\left|H_{0}\right|^{2}+\frac{2 c(m-1)}{m}<(m-1)\left|H_{0}\right|^{2},
$$

which is a contradiction. Hence $T=0$ and, using Theorem 2 in [11], this leads to the conclusion that $\Sigma^{m}$ is a cmc hypersurface in $M^{m+1}(c)$.

Finally, we observe that, using (4.19), we have

$$
|\phi|^{2} \leq 4 c+\frac{m}{m-1}|H|^{2}<r^{2},
$$

where $\phi$ is the traceless part of $\sigma$ and $r$ is the positive root of the polynomial

$$
P(t)=t^{2}+\frac{m(m-2)}{\sqrt{m(m-1)}}|H| t-m\left(c+|H|^{2}\right),
$$

and then, from Theorem 5 in [4], we get that $\phi=0$, which means that $\Sigma^{m}$ is totally umbilical in $M^{m+1}(c)$.

In the case of pmc surfaces, we can state the following two results.

Theorem 4.6. Let $\Sigma^{2}$ be a complete non-minimal pmc surface in $M^{n}(c) \times \mathbb{R}$, $n>2, c>0$, such that the angle between $H$ and $\xi$ is constant and

$$
|\sigma|^{2}+3 c|T|^{2} \leq 4|H|^{2}+2 c .
$$

Then, either

1. $\Sigma^{2}$ is pseudo-umbilical and lies in $M^{n}(c)$; or

2. $\Sigma^{2}$ is a torus $\mathbb{S}^{1}(r) \times \mathbb{S}^{1}\left(\sqrt{1 / c-r^{2}}\right)$ in $M^{3}(c)$, with $r^{2} \neq 1 /(2 c)$.

Proof. The map $p \in \Sigma^{2} \rightarrow\left(A_{H}-\mu \mathrm{I}\right)(p)$, where $\mu$ is a constant, is analytic, and, therefore, either $\Sigma^{2}$ is a pseudo-umbilical surface (at every point), or $H$ is an umbilical direction on a closed set without interior points. In the second case, $H$ is not an umbilical direction on an open dense set $W$. We shall work on this set and then we shall extend the results to the whole surface by continuity.

If $\Sigma^{2}$ is a pmc surface in $M^{n}(c) \times \mathbb{R}$, then either $\Sigma^{2}$ is pseudo-umbilical, i.e., $H$ is an umbilical direction everywhere, or, at any point in $W$, there exists a local orthonormal frame field that diagonalizes $A_{U}$ for any normal vector field $U$ defined on $W$ (see Lemma 1 in [3]). According to Theorem 1 in [3], if $\Sigma^{2}$ is a pseudoumbilical pmc surface in $M^{n}(c) \times \mathbb{R}$, then it lies in $M^{n}(c)$, and if the surface is not pseudo-umbilical, then it lies in $M^{4}(c) \times \mathbb{R}$.

In the following, we shall assume that $\Sigma^{2}$ is not pseudo-umbilical and we shall prove that, in this case, it is a torus in $M^{3}(c)$.

First, let $\left\{E_{3}=H /|H|, E_{4}, E_{5}\right\}$ be a local orthonormal frame field in the normal bundle, and then observe that $\left[A_{\alpha}, A_{\beta}\right]=0$ for all $\alpha$ and $\beta$. Moreover, diagonalizing simultaneously $A_{4}$ and $A_{5}$, we easily get

$$
\left(\operatorname{trace}\left(A_{4} A_{5}\right)\right)^{2}=2\left|A_{4}\right|^{2}\left|A_{5}\right|^{2} \leq \frac{1}{2}\left(\left|A_{4}\right|^{2}+\left|A_{5}\right|^{2}\right)^{2}=\frac{1}{2}|\mathcal{A}|^{4},
$$

which means that

$$
\operatorname{trace}\left[A_{4}, A_{5}\right]^{2}-\left(\operatorname{trace}\left(A_{4} A_{5}\right)\right)^{2}=-2\left|A_{4}\right|^{2}\left|A_{5}\right|^{2} \geq-\frac{1}{2}|\mathcal{A}|^{4} .
$$


Now, taking into account that

$$
\left|A_{\alpha} T\right|^{2}=\frac{1}{2}|T|^{2}\left|A_{\alpha}\right|^{2}
$$

for $\alpha \in\{4,5\}$, since trace $A_{\alpha}=0$, and then working exactly as in the proof of Theorem 4.4, we obtain

$$
\Delta|\mathcal{A}|^{2} \geq \frac{1}{2}|\mathcal{A}|^{4} \geq 0
$$

By hypothesis, we have that the Gaussian curvature $K$ of our surface satisfies

$$
2 K=2 c\left(1-|T|^{2}\right)+4|H|^{2}-|\sigma|^{2} \geq c|T|^{2} \geq 0,
$$

which means that $\Sigma^{2}$ is a parabolic space. Therefore, since $|\mathcal{A}|^{2}$ is a bounded subharmonic function, we get that $|\mathcal{A}|^{2}=0$, i.e., $A_{4}=A_{5}=0$. Moreover, using Proposition 3.4, we see that either $T=0$ or $T= \pm \xi$. Again as in Theorem 4.4 we discard the second case and we conclude that $\Sigma^{2}$ lies in $M^{3}(c)$ by using Theorem 2 in [11].

Finally, since $\Sigma^{2}$ is not pseudo-umbilical, from a result in [13] (see also Theorem 1.5 in [2]), we obtain that $|\sigma|^{2}=4|H|^{2}+2 c$ and that our surface is the torus $\mathbb{S}^{1}(r) \times \mathbb{S}^{1}\left(\sqrt{1 / c-r^{2}}\right)$, with $r^{2} \neq 1 /(2 c)$.

Theorem 4.7. Let $\Sigma^{2}$ be a complete non-minimal pmc surface in $M^{n}(c) \times \mathbb{R}$, $n>2, c<0$, such that $H$ is orthogonal to $\xi$ and

$$
|\sigma|^{2}+5 c|T|^{2} \leq 4|H|^{2}+4 c .
$$

Then $\Sigma^{2}$ is pseudo-umbilical and lies in $M^{n}(c)$.

Proof. Let us assume that $\Sigma^{2}$ is not pseudo-umbilical. Then, from (4.21), and working as in Theorem 4.5 we can prove that $\Sigma^{2}$ lies in $M^{3}(c)$. On the other hand, we observe that $|\sigma|^{2} \leq 4|H|^{2}+4 c<4|H|^{2}+2 c$, and, therefore, using a result in [19], we have that the surface is totally umbilical, which is a contradiction.

\section{References}

[1] Abresch, U. and Rosenberg, H.: A Hopf differential for constant mean curvature surfaces in $\mathbb{S}^{2} \times \mathbb{R}$ and $\mathbb{H}^{2} \times \mathbb{R}$. Acta Math. 193 (2004), no. 2, 141-174.

[2] Alencar, H. And do Carmo, M.: Hypersurfaces with constant mean curvature in spheres. Proc. Amer. Math. Soc. 120 (1994), no. 4, 1223-1229.

[3] Alencar, H., do Carmo, M. and Tribuzy, R.: A Hopf theorem for ambient spaces of dimensions higher than three. J. Differential Geom. 84 (2010), no. 1, 1-17.

[4] Alías, L. J. and García-Martínez, S. C.: On the scalar curvature of constant mean curvature hypersurfaces in space forms. J. Math. Anal. Appl. 363 (2010), no. 2, 579-587.

[5] Araújo, K. O. and Tenenblat, K.: On submanifolds with parallel mean curvature vector. Kodai Math. J. 32 (2009), no. 1, 59-76. 
[6] Batista, M.: Simons type equation in $\mathbb{S}^{2} \times \mathbb{R}$ and $\mathbb{H}^{2} \times \mathbb{R}$ and applications. Ann. Inst. Fourier (Grenoble) 61 (2011), no. 4, 1299-1322.

[7] Chen, B. Y. and Okumura, M.: Scalar curvature, inequality and submanifold. Proc. Amer. Math. Soc. 38 (1973), 605-608.

[8] Cheng, Q. M. And Nonaka, K.: Complete submanifolds in Euclidean spaces with parallel mean curvature vector. Manuscripta Math. 105 (2001), no. 3, 353-366.

[9] Colding, T. H. And Minicozzi II, W. P.: A course in minimal surfaces. Graduate Studies in Mathematics 121, American Mathematical Society, Providence, RI, 2011.

[10] ERbacher, J.: Isometric immersions of constant mean curvature and triviality of the normal connection. Nagoya Math. J. 45 (1972), 139-165.

[11] Eschenburg, J. H. And Tribuzy, R.: Existence and uniqueness of maps into affine homogeneous spaces. Rend. Sem. Mat. Univ. Padova 89 (1993), 11-18.

[12] Fetcu, D., Oniciuc, C. And Rosenberg, H.: Biharmonic submanifolds with parallel mean curvature in $\mathbb{S}^{n} \times \mathbb{R}$. J. Geom. Anal. 23 (2013), no. 4, 2158-2176.

[13] Hoffman, D. A.: Surfaces of constant mean curvature in manifolds of constant curvature. J. Differential Geometry 8 (1973), 161-176.

[14] Li, A. M. AND Li, J. M.: An intrinsic rigidity theorem for minimal submanifolds in a sphere. Arch. Math. (Basel) 58 (1992), no. 6, 582-594.

[15] Lu, Z.: Normal scalar curvature conjecture and its applications. J. Funct. Anal. 261 (2011), no. 5, 1284-1308.

[16] Nomizu, K. And Smyth, B.: A formula of Simons' type and hypersurfaces with constant mean curvature. J. Differential Geometry 3 (1969), 367-377.

[17] Santos, W.: Submanifolds with parallel mean curvature vector in spheres. Tôhoku Math. J. (2) 46 (1994), no. 3, 403-415.

[18] Simons, J.: Minimal varieties in Riemannian manifolds. Ann. of Math. (2) 88 (1968), 62-105.

[19] Tribuzy, R.: Hopf's method and deformations of surfaces preserving mean curvature. An. Acad. Brasil. Cienc. 50 (1978), no. 4, 447-450.

[20] Yau, S.-T.: Submanifolds with constant mean curvature. I. Amer. J. Math. 96 (1974), 346-366.

[21] YAU, S.-T.: Harmonic functions on complete Riemannian manifolds. Comm. Pure. Appl. Math. 28 (1975), 201-228.

Received December 27, 2011.

Dorel Fetcu: Department of Mathematics and Informatics, Gheorghe Asachi Technical University of Iaşi, 11 Carol I Blvd., 700506 Iaşi, Romania.

E-mail: dfetcu@math.tuiasi.ro; dorel@impa.br

Harold Rosenberg: IMPA, Estrada Dona Castorina 110, 22460-320 Rio de Janeiro, Brazil.

E-mail: rosen@impa.br

The first author was partially supported by a grant of the Romanian National Authority for Scientific Research, CNCS-UEFISCDI, project number PN-II-RU-TE-2011-3-0108. 